\title{
STUDY ON PREVALENCE OF METHICILLIN RESISTANT STAPHYLOCOCCUS AUREUS; COMPARISON OF FOUR DIAGNOSTIC METHODS FOR DETECTING MRSA- FROM A TERTIARY CARE HOSPITAL, MADURAI
} Rajendran Thiruvannamalai ${ }^{1}, J^{2}$ ansi Charles², Raja Sundaramurthy33, Ramesh Arunagiri, Vithiya Ganesan ${ }^{5}$,
Geni Veerathevar German Soundaram ${ }^{6}$, Brindha Vetrinallathambi ${ }^{7}$ 1Assistant Professor, Department of Microbiology, Velammal Medical College Hospital and Research Institute (VMCH\&RI).
2Professor and HOD, Department of Microbiology, Velammal Medical College Hospital and Research Institute (VMCH\&RI).
3Assistant Professor, Department of Microbiology, Velammal Medical College Hospital and Research Institute (VMCH\&RI).
${ }^{4}$ Associate Professor, Department of Microbiology, Velammal Medical College Hospital and Research Institute (VMCH\&RI).
${ }^{5}$ Assistant Professor, Department of Microbiology, Velammal Medical College Hospital and Research Institute (VMCH\&RI).
${ }^{6}$ Assistant Professor, Department of Microbiology, Velammal Medical College Hospital and Research Institute (VMCH\&RI).
${ }^{7}$ Assistant Professor, Department of Microbiology, Velammal Medical College Hospital and Research Institute (VMCH\&RI).

\section{ABSTRACT}

\section{BACKGROUND}

Prevalence of Methicillin-Resistant Staphylococcus aureus (MRSA) in India ranges from 30 to $70 \%$ which leads to high mortality, increased economic burden and high treatment failure when compared to MSSA. Rapid and accurate detection of MRSA is essential to take measures for infection control and also to prevent unnecessary use of antibiotics.

\section{MATERIALS AND METHODS}

Prospective observational study was carried out in our tertiary care hospital over a period of 6 months (May-October 2017). All Staphylococcus grown among 240 various clinical samples received were tested for MRSA by four phenotypic methods - cefoxitin and oxacillin disc diffusion test and MIC oxacillin E test and oxacillin resistant screen agar test (ORSA) with mecA-PCR as gold standard.

\section{RESULTS}

A total of 103 out of 200 (51.5\%) bacterial isolates were Staphylococcus aureus. Pus from diabetic wound yielded high number of growth (42\%) followed by blood (36\%) which is alarming note. $55.4 \%$ were identified as MRSAs by PCR; most common presenting group was $>50$ years $(36.8 \%)$ followed by infants (28\%). Cefoxitin disc method has the highest sensitivity and specificity of $98.2 \%$ \& $100 \%$ followed by E test $(94.2 \%$ \& $95.8 \%)$ and ORSA (87.5\% \& 94.2\%). Oxacillin disc diffusion method had the least $79.5 \%$ \& 92.9\%. Most MRSA isolates were multi drug resistant.

\section{CONCLUSION}

Cefoxitin disc diffusion when combined with Oxacillin agar or E test will be helpful to detect all MRSA strains including hyper production of betalactamases. As these phenotypic assays are simple and relatively cheap, this can be used as an alternative to PCR in resource constraint settings.

\section{KEYWORDS}

MRSA; Prevalence; mecA Gene; Cefoxitin Disc; Phenotypic Tests.

HOW TO CITE THIS ARTICLE: Thiruvannamalai R, Charles J, Sundaramurthy R, et al. Study on prevalence of methicillin resistant staphylococcus aureus; comparison of four diagnostic methods for detecting MRSA-from a tertiary care hospital, Madurai. J. Evolution Med. Dent. Sci. 2018;7(07):864-868, DOI: 10.14260/jemds/2018/197

\section{BACKGROUND}

Methicillin-Resistant Staphylococcus aureus (MRSA) is an important cause of nosocomial and community associated infections throughout the world.(1) First MRSA was described among nosocomial isolates of Staphylococcus aureus in 1961, England which subsequently spread throughout the world.(2) In India the prevalence of MRSA ranges from 30 to $70 \%$ which leads to high mortality, increase economic burden. ${ }^{(3,4)}$

'Financial or Other Competing Interest': None.

Submission 04-01-2018, Peer Review 01-02-2018,

Acceptance 07-02-2018, Published 12-02-2018.

Corresponding Author:

Dr. Jhansi Charles,

Professor and HOD, Department of Microbiology,

Velammal Medical College Hospital and

Research Institute (VMCH\&RI), Anuppanadi,

Madurai-625009, Tamilnadu.

E-mail: Jhansi_charles@yahoo.co.in

DOI: $10.14260 /$ jemds $/ 2018 / 197$

\section{(c) $\frac{10}{\mathrm{BV}}(\mathrm{Sc})$}

MRSA treatment will be more problematic as these strains often show resistance to wide range of antibiotics compared to methicillin susceptible isolates.(3) Therefore, rapid and accurate detection of MRSA is very essential in order to choose appropriate therapy, to take necessary measures for infection control and also to prevent unnecessary use of glycol-peptides antibiotics.

MRSA strains harbour the mecA gene, which encodes a penicillin binding protein (PBP2a) with low affinity for all $ß$ lactam antibiotics including methicillin which has limited therapeutic option.(5) Hence methods used to identify MRSA from clinical samples should have high sensitivity and specificity and most importantly the result should be available within a short time. Different phenotypic methods such as oxacillin and cefoxitin disc diffusion test, oxacillin agar screening test, and determination of minimum inhibitory concentration (MIC) for oxacillin and cefoxitin are available in clinical laboratories.(6-9) Phenotypic expression is affected by various conditions such as temperature, 
osmolarity of the medium and inoculum size that may affect the accuracy of the methodsused to detect methicillin resistance. $(9,10)$ Some strains of S.aureus hyper produce beta lactamase known as Borderline Oxacillin Resistant $S$. aureus (BORSA) will be oxacillin resistant, do not possess the usual genetic mechanism for MRSA resistance.(8)

Even though genotypic method which detects the mecA gene to identify MRSA among the S.aureus isolates is considered to be the gold standard test for detecting MRSA, $(6,7,10)$ it could not be adopted as a routine method of screening MRSA in small laboratories since it requires costlier and sophisticated equipment. Hence the easily available, non- expensive phenotypic methods are the one needed to identify the MRSA isolates for many labs.

Our study is planned to know the prevalence of MRSA among different clinical samples received in laboratory with the aim to compare the PCR of the mecA gene with four phenotypic methods - cefoxitin disc diffusion test, oxacillin disc diffusion test and MIC of oxacillin by E test and screen agar test for detection of MRSA.

\section{MATERIALS AND METHODS}

Prospective observational study was carried out in Velammal Medical College Hospital and Research Institute, Madurai, Tamilnadu over a period of 6 months (May -October 2017).

A total of 240 samples received in the lab during the study period were tested for Staphylococcus aureus from various clinical samples like blood, pus, urine, sputum and throat swab. Isolates were identified as $S$. aureus by their colony morphology, gram staining, catalase and coagulase tests (both tube and slide coagulase tests).

All the Staphylococcal isolates were tested for antibiotic susceptibility by the Kirby-Bauer disc diffusion method. Following antibiotics were selected based on clinical and laboratory standards Institute (CLSI) guideline: Penicillin (10 u), Erythromycin $(15 \mu \mathrm{g})$, Clindamycin $(2 \mu \mathrm{g})$, Ciprofloxacin (5 $\mu \mathrm{g})$, Cotrimoxazole (1.25/23.75 ug), Amikacin (30 $\mu \mathrm{g})$, Oxacillin $(1 \mu \mathrm{g})$, Cefoxitin $(30 \mu \mathrm{g})$ and Linezolid $(30 \mu \mathrm{g})$, Vancomycin E strip. Antibiotic discs and E strips were bought from Hi-Media diagnostic laboratory, Mumbai.(11)

\section{Study Design}

Phenotype Identification of MRSA:

Phenotypic methods for detection of MRSA strains were carried out according to CLSI guideline as follows:

\section{Cefoxitin Disc Diffusion Test}

Cefoxitin $(30 \mu \mathrm{g})$ disc diffusion method was carried out on Mueller-Hinton agar (MHA) by using a $30 \mu \mathrm{g}$ cefoxitin disc. The standardised inoculum was streaked on MHA and incubated at $37^{\circ} \mathrm{C}$ for $24 \mathrm{hrs}$. An inhibition zone diameter of $\leq 21 \mathrm{~mm}$ was reported as methicillin resistant and a diameter of $\geq 22 \mathrm{~mm}$ was considered as methicillin sensitive

\section{Oxacillin Disc Diffusion Test}

Oxacillin $(1 \mu \mathrm{g})$ disc diffusion method was carried out on Mueller-Hinton agar supplemented with $4 \% \mathrm{NaCl}$. Plates were incubated at $35^{\circ} \mathrm{C}$ for $24 \mathrm{hrs}$. The isolates were considered as resistant when the diameter of inhibition zone of oxacillin was $\leq 10 \mathrm{~mm}$, as intermediate when the diameter was $11-12 \mathrm{~mm}$ and as sensitive when the diameter was $\geq 13$ $\mathrm{mm}$.

\section{Oxacillin E Strip Test}

In Muller-Hinton agar plates supplemented with $4 \% \mathrm{NaCl}$, the test strain was lawn cultured, oxacillin E strip was placed on the medium and incubated for $24 \mathrm{~h}$ at $35^{\circ} \mathrm{C}$. After incubation, inhibitory concentration studied as zone of inhibition intersected the strip in the form of ellipse and interpreted according to the CLSI criteria. MIC of $\leq 2 \mu \mathrm{g} / \mathrm{ml}$ was considered as sensitive; $\geq 4 \mu \mathrm{g} / \mathrm{ml}$ was considered as resistant.

\section{Oxacillin Resistant Screen Agar Test (ORSA)}

Muller-Hinton agar plates containing $4 \% \mathrm{NaCl}, 6 \mu \mathrm{g} / \mathrm{ml}$ of oxacillin and chromogenic component aniline dye were prepared. Agar base were obtained from Himedia, Mumbai. Aloopful of $0.5 \mathrm{McF}$ arland's suspension of the isolate was inoculated as a spot on the agar surface and it was incubated at $35^{\circ} \mathrm{C}$ for $24 \mathrm{~h}$. The plates were observed for colour change from original grey to blue to identify as MRSA.

\section{Genotype Identification of MRSA by PCR}

Genotypic identification of mecA gene was carried out by conventional Polymerase Chain Reaction (PCR) using thermocycler in Helini Biomolecules, Chennai. DNA was extracted by the rapid cell lysis method using Helini extraction kit- $1.5 \mathrm{~mL}$ of an overnight culture of bacteria grown in Mueller-Hinton broth was harvested by centrifuging in a micro centrifuge tube at 10, $000 \mathrm{rpm}$ for 5 mins. $400 \mu \mathrm{L}$ of lysis buffer with $40 \mu \mathrm{L}$ of proteinase $\mathrm{K}$ was added to the pellet and incubated at $70^{\circ} \mathrm{C}$ for 10 minutes. After adding 100 $\mu \mathrm{L}$ of isopropanol, the entire sample was pipetted into pure fast spin column and centrifuged for $1 \mathrm{~min}$. Flow through was discarded and spin column washed with $500 \mu \mathrm{L}$ wash buffer $1 \& 2$ and then $100 \mu \mathrm{L}$ of pre-warmed buffer EB added and incubated for 2 mins. After the final centrifugation extracted bacterial DNA was collected for PCR assay.

PCR for mecA gene was performed using the following primers- Forward (5' GCA ATC GCT AAA GAA CTA AG 3') and Reverse (5'GGG ACC AAC ATA ACC TAA 3') primers in the thermocycler (Eppendorf Master Cycler Gradient thermocycler, Germany) with a final reaction mixture volume of $50 \mu$ l.Cycling conditions were $94^{\circ} \mathrm{C}-3 \mathrm{mins}$ of initial denaturation followed by 30 cycles of Denaturation at $94^{\circ} \mathrm{C}-1 \mathrm{~min}$, Annealing at $60^{\circ} \mathrm{C}-1 \mathrm{~min}$, Extension at $72^{\circ} \mathrm{C}-$ $1 \mathrm{~min}$ and a final extension of $72^{\circ} \mathrm{C}-5 \mathrm{mins}$. The amplicon for mecA gene was detected by the band at $220 \mathrm{bp}$ on an agarose gel $(2 \%)$ with ethidium bromide by electrophoresis.

\section{Statistical Analysis}

Data analysis was done using SPSS 16 version. Comparison of all phenotypic methods were carried out taking mecA PCR as a gold standard technique, sensitivity and specificity were expressed as percentage using Chi square test. Prevalence, Age, sex and risk factors analysis were expressed as percentage.

\section{RESULTS}

Among the 240 samples received during the study period, 200 samples showed growth. Total of 240 isolates were detected from those 200 samples. A total of 103 samples out of 200 (51.5\%) yielded the growth of Staphylococcus aureus during the study period. Pus sample $(40 / 103=38.8 \%)$ followed by blood sample $(37 / 103=35.9 \%)$ yielded the highest number of S.aureus growth (Figure 1). 
Out of 103 S.aureus isolates 57(55.4\%) were Methicillin Resistant S.aureus (MRSA) and 46 (44.6\%) were Methicillin Sensitive S.aureus (MSSA) as detected by taking PCR as a gold standard technique. Sample wise analysis of MRSA showed that 29 out of $40(72.5 \%)$ S.aureus strains isolated from pus followed by 19 out of 37(51.4\%) from blood were MRSA (Table 1).

Age wise distribution analysis revealed that MRSA were most common among the older age group (21/57=36.8\%) followed by infants (28\%) (Figure 2).

Among the 57 MRSA isolates $16(28.06 \%)$ were associated with risk factors. Analysis of risk factors revealed that 10 patients (17.54\%) of MRSA wound infection had diabetes; 3 patients $(5.26 \%)$ had chronic liver disease and 2 patients (3.5\%) had a history of intermittent catheterisation; 1 patient $(1.75 \%)$ had a history of prolonged self-medication with Quinolone antibiotic.

All the 103 S.aureus isolates were subjected to PCR to identify mecA gene. Among these 57 (55.4\%) were identified as MRSA by PCR.

All the 103 S.aureus isolates were also subjected to methicillin susceptibility testing by disc diffusion method by using $30 \mu \mathrm{g}$ Cefoxitin and $1 \mu \mathrm{g}$ Oxacillin discs, E-Test (oxacillin), Oxacillin Resistant Screen Agar. Cefoxitin disc diffusion method identified 56 (54.4\%) isolates of S.aureus as MRSA followed by Oxacillin E-Test identified 54 (52.4\%) S.aureus strains as MRSA (Table 2).

Comparison of all phenotypic methods were carried out taking PCR as a gold standard technique. Among the 57 PCR positive MRSA strains, Cefoxitin disc identified one isolate as negative (false negative) (Table 3 ).

Cefoxitin disc method has the highest sensitivity (98.2\%) and specificity (100\%) and the PPV and NPV are $98.24 \%$ and $100 \%$ respectively (Table 4 ).

Most of our MRSA isolates were multi drug resistant (MDR) as $91 \%$ resistant to fluoroquinolones, $88 \%$ resistant to aminoglycosides, $83 \%$ cotrimoxazole (Table 5).

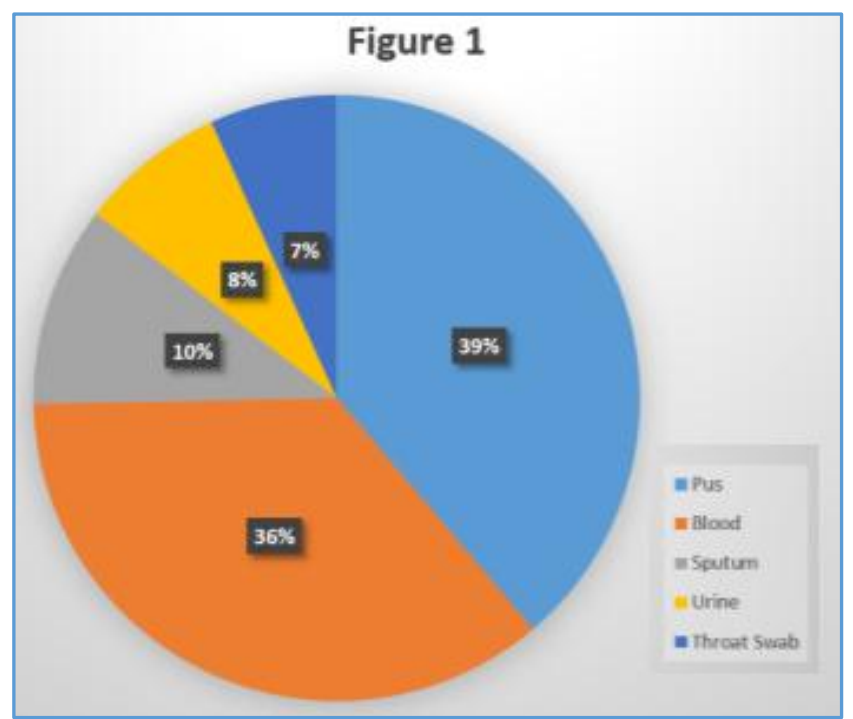

Figure 1. Staphylococcus aureus isolates among various clinical samples

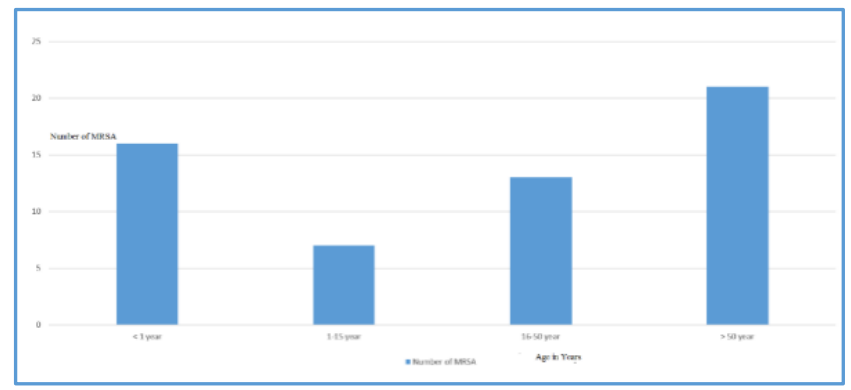

Figure 2. Staphylococcus aureus isolates among various age groups

\begin{tabular}{|c|c|c|c|}
\hline Specimen & S.aureus & MRSA & Percentage \\
\hline Pus & 40 & 29 & $72.5 \%$ \\
\hline Blood & 37 & 19 & $51.4 \%$ \\
\hline Sputum & 11 & 4 & $36.4 \%$ \\
\hline Throat swab & 8 & 2 & $25 \%$ \\
\hline Urine & 7 & 3 & $42.91 \%$ \\
\hline Total & 103 & $\mathbf{5 7}$ & $\mathbf{5 5 . 3 \%}$ \\
\hline Table 1. Sample wise distribution of Methicillin Resistant \\
Staphylococcus aureus (MRSA) \\
\hline
\end{tabular}

\begin{tabular}{|c|c|c|}
\hline Test (n=103) & MRSA & MSSA \\
\hline PCR (mecA gene) & $57(55.4 \%)$ & $46(44.6 \%)$ \\
\hline Cefoxitin Disc & $56(54.36 \%)$ & $47(45.63 \%)$ \\
\hline E-Test & $54(52.42 \%)$ & $49(47.57 \%)$ \\
\hline ORSA & $51(49.51 \%)$ & $52(50.48 \%)$ \\
\hline Oxacillin Disc & $48(46.6 \%)$ & $55(53.39 \%)$ \\
\hline
\end{tabular}

Table 2. Detection of Methicillin Resistant Staphylococcus aureus (MRSA) by various phenotypic and genotypic method

\begin{tabular}{|c|c|c|c|}
\hline Methods & $\begin{array}{c}\text { True } \\
\text { Positives }\end{array}$ & $\begin{array}{c}\text { False } \\
\text { Negatives }\end{array}$ & $\begin{array}{c}\text { False } \\
\text { Positives }\end{array}$ \\
\hline PCR & 57 & - & - \\
\hline Cefoxitin disc & 55 & 1 & - \\
\hline E-Test & 49 & 3 & 2 \\
\hline ORSA & 42 & 6 & 3 \\
\hline Oxacillin disc & 35 & 9 & 4 \\
\hline \multicolumn{4}{|r|}{ Table 3. Comparison of PCR for mecA gene } \\
with phenotypic methods \\
\hline
\end{tabular}

\begin{tabular}{|c|c|c|c|c|}
\hline TEST & Sensitivity & Specificity & $\begin{array}{c}\text { Positive } \\
\text { Predictive } \\
\text { Value }\end{array}$ & $\begin{array}{c}\text { Negative } \\
\text { Predictive } \\
\text { Value }\end{array}$ \\
\hline $\begin{array}{c}\text { Cefoxitin } \\
\text { disc }\end{array}$ & $98.2 \%$ & $100 \%$ & $98.24 \%$ & $100 \%$ \\
\hline E-Test & $94.2 \%$ & $95.8 \%$ & $96.42 \%$ & $94.23 \%$ \\
\hline ORSA & $87.5 \%$ & $94.2 \%$ & $92.44 \%$ & $90.22 \%$ \\
\hline $\begin{array}{c}\text { Oxacillin } \\
\text { disc }\end{array}$ & $79.5 \%$ & $92.9 \%$ & $88.30 \%$ & $82.93 \%$ \\
\hline \multicolumn{4}{|c|}{ Table 4. Sensitivity and Specificity of various } \\
phenotypic tests \\
\hline
\end{tabular}

\begin{tabular}{|c|c|c|c|c|c|c|c|c|}
\hline & $\mathrm{P}$ & $\mathrm{CD}$ & $\mathrm{E}$ & $\mathrm{CIP}$ & $\mathrm{AK}$ & $\mathrm{COT}$ & $\mathrm{VA}$ & $\mathrm{LZ}$ \\
\hline $\mathrm{MRSA}$ & 57 & 32 & 37 & 52 & 50 & 47 & 0 & 0 \\
$\mathrm{~N}=57$ & $(100 \%)$ & $(56.1 \%)$ & $(64.9 \%)$ & $(91.2 \%)$ & $(87.7 \%)$ & $(82.5 \%)$ & - & - \\
\hline
\end{tabular}

Table 5. Other antibiotic resistance pattern of MRSA isolates

P-Penicillin (10 u), CD- Clindamycin (2 $\mu \mathrm{g}), \quad$ EErythromycin (15 $\mu \mathrm{g})$, CIP-Ciprofloxacin (5 $\mu \mathrm{g})$, AK- Amikacin $(30 \mu \mathrm{g})$, COT- Cotrimoxazole $(1.25 / 23.75 \mathrm{ug})$, VAVancomycin E strip, LZ-Linezolid $(30 \mu \mathrm{g})$. 


\section{DISCUSSION}

For the past 50 years, MRSA is probably the most challenging bacterial pathogen causing significant morbidity and mortality that affect patients in hospital as well as in the community.(12) Methicillin-resistant strains in all over the world have been posing a great difficulty in selecting antimicrobial agents for the management of the infections that leads to treatment failure. Hence, an accurate and rapid detection of methicillin resistance in Staphylococci is therefore important, not only for choosing the appropriate antibiotic therapy, but also to control of the endemicity of the MRSA. $(2,3)$

In our study, $51.5 \%(103 / 200)$ of the isolates were Staphylococcus aureus which was isolated as the prime organism in all infections except urinary tract infection. Occurrence of S.aureus as the prime organism may be due to the presence of a number of enzymes and toxins produced by S.aureus which may inhibit the phagocytic and opsonic mechanisms. ${ }^{(1,12)}$ Less frequent occurrence of S.aureus in UTI may be due to genotypic factors of S.aureus which may affect the uroepithelial susceptibility to the adherence molecules.(13)

Our study revealed S.aureus was the common isolate from pus samples from localised diabetic wound lesions(39\%) which was in concordance with the findings of dechen $\mathrm{C}$ Tseringet al.,(14) who also reported $42 \%$ of isolation from pus samples. Localisation of pus is mainly due to the unique presence of coagulase in S.aureus which has the capacity of forming a wall of fibrin clot around the lesion. The challenging thing is nearly $36 \%$ of S.aureus was isolated from blood which was even higher than the other studies. $(15,16)$ Bacteraemia due to S.aureus has increased dramatically in recent years which may end up in life-threatening complications like infective endocarditis and metastatic infections. This is driving as urgent need for improved strategies to prevent these infections.

In our study $55.4 \%$ were MRSAs which was much higher than other studies, $(3,17,18)$ which indicates MRSA is steadily increasing which may be due to the adverse use of antibiotics or poor infection control practices in the environment. Among MRSA, 28.15\% were isolated from pus samples of diabetic wound infection which is in concordance with finding of sgangaet al.,(19) reported $30 \%$ of MRSA. The increased incidence of MRSA in wound infection may be due to the production on Panton Valentine Leucocidin by MRSA which is shown too associated with tissue necrosis.

Age wise analysis revealed MRSA was commonly present in the extremes of age which is similar to the other study results.(20,21) Higher incidence in older age group may be due to immunosuppression caused by various factors like diabetes mellitus, chronic hepatitis, anaemia and nonadherence to antibiotic course and infants there may be poor development of immune system which may associated with acquired chemotactic or opsonic defects

Early and accurate determination of Methicillin resistance is of key importance in the prognosis of infections caused by S.aureus. Although many phenotypic methods of detection of this resistance have been developed they often show insufficient sensitivity and specificity to ensure appropriate treatment of the MRSA infected patients. $(1,8,13)$ The sensitivity and specificity of these methods vary depending on the patients carrying them, environmental factors, and the techniques used.
Our study revealed disc diffusion using cefoxitin disc showed higher sensitivity (98.2\%) and specificity (100\%) which is almost similar to the study results of Pourmand et al.,(9) who also documented $99.1 \%$ sensitivity and $98.1 \%$ specificity by cefoxitin disc method. Even Swenson et al., Jain et al.,(22,23) showed $100 \%$ specificity and sensitivity with cefoxitin disc methods. Our findings also emphasis that cefoxitin disc method is close enough to PCR in identifying MRSA.

Oxacillin disc diffusion method had the least sensitivity (79.5\%) and specificity (92.9\%) followed by ORSA $87.5 \%$ sensitivity and $94.2 \%$ specificity. E test had better sensitivity and specificity (94.2\% \& 95.8\%) when compare to ORSA and oxacillin disc but inferior to cefoxitin disc diffusion test. This may be due to the fact that detection of MRSA by Cefoxitin based methods will not be affected by temperature variations between $35^{\circ} \mathrm{C}$ and $37^{\circ} \mathrm{C}$ but Oxacillin method will be affected by temperature variations especially if it is increased above $35^{\circ} \mathrm{C}$ and also medium supplementations. Similar factors decrease the sensitivity and specificity of ORSA and E-Test.(24)

False positivity (resistant to oxacillin but sensitive to cefoxitin and negative for mecA gene ) in ORSA methods and E-Test is due to the hyper production of betalactamase which may lead to phenotypic expression of oxacillin resistance do not possess the usual genetic mechanism for such resistance. Probably these strains under antibiotic pressure may evolve into fully resistant isolates subsequently.(25)

One more problematic fact is most of the MRSA isolates were multi drug resistant (MDR). Isolates have shown 91\% resistant to fluoroquinolones, $88 \%$ resistant to aminoglycosides, $83 \%$ cotrimoxazole and more than $50 \%$ to macrolides. This is an alarming note as only few therapeutic opinions available. So early diagnosis is will be helpful to avoid the unnecessary use of antibiotics.

\section{CONCLUSION}

Our study findings reveal that oxacillin disk diffusion method was found to be less sensitive for the detection of MRSA. Cefoxitin disc diffusion followed be Oxacillin E strip was in concordance with the results of PCR for mecA gene. As cefoxitin method is easy to perform, does not require special technique, media preparation, incubation temperature and more cost effective in comparison to other methods this can be used as an alternative to PCR for the detection of MRSA in resource constraint settings. Cefoxitin when combined with Oxacillin agar or E test will be helpful to determine the all MRSA strains including hyper production of betalactamase.

\section{REFERENCES}

[1] Stefani S, Chung DR, Lindsay JA, et al. Meticillinresistant Staphylococcus aureus (MRSA): global epidemiology and harmonisation of typing methods. Int J Antimicrob Agents 2012;39(4):273-82.

[2] Brown DF. Detection of methicillin/oxacillin resistance in staphylococci. J Antimicrob Chemother 2001;48 (Suppl 1):65-70.

[3] Rajaduraipandi K, Mani KR, Panneerselvam K, et al. Prevalence and antimicrobial susceptibility pattern of methicillin resistant Staphylococcus aureus: a multicentre study. Indian J Med Microbiol 2006;24(1):34-8. 
[4] Saikia L, Nath R, Choudhury B, et al. Prevalence and antimicrobial susceptibility pattern of methicillinresistant Staphylococcus aureus in Assam. Indian J Crit Care Med 2009;13(3):156-8.

[5] Hartman BJ, Tomasz A. Low-affinity penicillin-binding protein associated with beta-lactam resistance in Staphylococcus aureus. J Bacteriol 1984;158(2):513-6.

[6] Gradelski E, Valera L, Aleksunes L, et al. Correlation between genotype and phenotypic categorization of Staphylococci based on methicillin susceptibility and resistance. J Clin Microbiol 2001;39(8):2961-3.

[7] Koupahi H, Jahromy HS, Rahbar M. Evaluation of different phenotypic and genotypic methods for detection of Methicillin Resistant Staphylococcus aureus (MRSA). Iran J Pathol 2016;11(4):370-6.

[8] Farahani A, Mohajeri P, Gholamine B, et al. Comparison of different phenotypic and genotypic methods for the detection of Methicillin-Resistant Staphylococcus Aureus. North Am J Med Sci 2013;5(11):637-40.

[9] Pourmand MR, Hassanzadeh S, Mashhadi R, et al. Comparison of four diagnostic methods for detection of methicillin resistant Staphylococcus aureus. Iran J Microbiol 2014;6(5):341-4.

[10] Baddour MM, AbuElKheir MM, Fatani AJ. Comparison of mecA polymerase chain reaction with phenotypic methods for the detection of methicillin-resistant Staphylococcus aureus. Curr Microbiol 2007;55(6):473-9.

[11] Clinical Laboratory Standards Institute. New Antimicrobial Susceptibility Testing Standards - CLSI http://clsi.org/blog/2015/01/08/clsi-publishes-newantimicrobial-susceptibility-testing-standards/

[12] Vidhani S, Mehndiratta PL, Mathur MD. Study of methicillin resistant S. aureus (MRSA) isolates from high risk patients. Indian J Med Microbiol 2001;19(2):13-6.

[13] Mohanasoundaram KM, Lalitha MK. Comparison of phenotypic versus genotypic methods in the detection of methicillin resistance in Staphylococcus aureus. Indian J Med Res 2008;127(1):78-84.

[14] Tsering DC, Pal R, Kar S. Methicillin-Resistant Staphylococcus Aureus: prevalence and current susceptibility pattern in Sikkim. J Glob Infect Dis 2011;3(1):9-13.
[15] Naber CK. Staphylococcus aureus bacteremia: epidemiology, pathophysiology and management strategies. Clin Infect Dis 2009;48 (Suppl 4):S231-S7.

[16] Vijayamohan N, Nair SP. A study of the prevalence of methicillin-resistant Staphylococcus aureus in dermatology inpatients. Indian Dermatol Online J 2014;5(4):441-5.

[17] Saxena S, Singh K, Talwar V. Methicillin-resistant Staphylococcus aureus prevalence in community in the east Delhi area. Jpn J Infect Dis 2003;56(2):54-6.

[18] Pillai MM, Latha R, Sarkar G. Detection of methicillin resistance in staphylococcus aureus by polymerase chain reaction and conventional methods: a comparative study. J Lab Physicians 2012;4(2):83-8.

[19] Sganga G, Tascini C, Sozio E, et al. Early recognition of methicillin-resistant Staphylococcus aureus surgical site infections using risk and protective factors identified by a group of Italian surgeons through Delphi method. World J Emerg Surg 2017;12:25.

[20] Madani TA. Epidemiology and clinical features of methicillin-resistant Staphylococcus aureus in the University Hospital, Jeddah, Saudi Arabia. Can J Infect Dis 2002;13(4):245-50.

[21] Sangvik M, Olsen RS, Olsen K, et al. Age-and genderassociated Staphylococcus aureus spa types found among nasal carriers in a general population: the Tromsø Staph and Skin study. J Clin Microbiol 2011;49(12):4213-8.

[22] Swenson JM, Tenover FC, CDS Group. Results of disk diffusion testing with cefoxitin correlate with presence of meca in Staphylococcus spp. J Clin Microbiol 2005;43(8):3818-23.

[23] Jain A, Agarwal A, Verma RK. Cefoxitin disc diffusion test for detection of meticillin-resistant staphylococci. J Med Microbiol 2008;57(Pt 8):957-61.

[24] Broekema NM, Van TT, Monson TA, et al. Comparison of cefoxitin and oxacillin disk diffusion methods for detection of meca-mediated resistance in Staphylococcus aureus in a Large-Scale Study. J Clin Microbiol 2009;47(1):217-9.

[25] Maalej SM, Rhimi FM, Fines M, et al. Analysis of Borderline Oxacillin-Resistant Staphylococcus aureus (BORSA) strains isolated in Tunisia. J Clin Microbiol 2012;50(10):3345-8. 